coronaviruses. ${ }^{6}$ When it comes to wearing masks, a simple nonpharmaceutical intervention method with minimal side effects, how did the lack of evidence lead to recommendations against wearing them among the general public at the beginning of the pandemic? And even later, the CDC only recommended wearing masks to prevent asymptotic carriers and presymptomatic patients from spreading the virus. ${ }^{7}$

Again, we remind ourselves, when issues of public health are concerned, we must question whether the absence of evidence is a valid justification for inaction. ${ }^{8}$ Statements about the absence of evidence are common, such as protective effects of masks for the general public at the beginning of current COVID-19 pandemic. However, can we be comfortable that the absence of solid and clear evidence is equivalent to the position that masks provide no protective effects or only negligible effects? For this global threat, it is better to be safe than sorry, and we should take every possible reasonable intervention.

Financial support. No financial support was provided relevant to this article. Conflicts of interest. All authors report no conflicts of interest relevant to this article.

\section{References}

1. MacIntyre CR, Wang Q. Physical distancing, face masks, and eye protection for prevention of COVID-19. Lancet 2020;395:1950-1951.

2. Scientific brief: community use of cloth masks to control the spread of SARSCoV-2. Centers for Disease Control and Prevention website. https://www. cdc.gov/coronavirus/2019-ncov/more/masking-science-sars-cov2.html. Published 2020. Accessed February 3, 2021.

3. Understanding the difference. Centers for Disease Control and Prevention website. https://www.cdc.gov/niosh/npptl/pdfs/UnderstandDifference Infographic-508.pdf. Published 2020. Accessed February 3, 2021.

4. Alderson P. Absence of evidence is not evidence of absence. BMJ 2004;328:476-477.

5. Liang M, Gao L, Cheng C, et al. Efficacy of face mask in preventing respiratory virus transmission: A systematic review and meta-analysis. Travel Med Infect Dis 2020;36:101751.

6. Beigel JH, Tomashek KM, Dodd LE, et al. Remdesivir for the treatment of COVID-19- final report. N Engl J Med 2020. doi: 10.1056/NEJMoa2007764.

7. Federal Register notice: wearing of face masks while on conveyances and at transportation hubs. Centers for Disease Control and Prevention website. https://www.cdc.gov/quarantine/masks/mask-travel-guidance. html. Published 2020. Accessed February 3, 2021.

8. Altman DG, Bland JM. Absence of evidence is not evidence of absence. BMJ 1995;311:485.

\title{
Indirect transmission of severe acute respiratory syndrome coronavirus virus 2 (SARS-CoV-2): What do we know and what do we not know?
}

\author{
M. Khalid Ijaz DVM, MSc(Hons), PhD, FRSPH ${ }^{1,2}$, Raymond W. Nims PhD $^{3}$ (1) and Julie McKinney PhD ${ }^{1}$ \\ ${ }^{1}$ Global Research and Development for Lysol and Dettol, Reckitt Benckiser, LLC, Montvale, New Jersey, ${ }^{2}$ Department of Biology, Medgar Evers College, City \\ University of New York (CUNY), Brooklyn, New York and ${ }^{3}$ RMC Pharmaceutical Solutions, Longmont, Colorado
}

To the Editor-We wish to point out that 12 months into the coronavirus disease 2019 (COVID-19) pandemic, with >111 million reported cases and $>2.4$ million deaths, many knowledge gaps still need to be resolved empirically to fully appreciate the risks associated with high-touch environmental surface (HITES) contamination. It has been argued that indirect transmission through contaminated HITES is an unlikely route of transmission for severe acute respiratory syndrome coronavirus virus 2 (SARS-CoV-2) (eg, Goldman ${ }^{1}$ and Meyerowitz et $\mathrm{al}^{2}$ ) Why? Is this position based on data, and if so what data?

The World Health Organization ${ }^{3}$ rightly has made the point that it is difficult to separate potential direct and indirect exposure in establishing transmission relevancy. The safest approach is, therefore, to avoid discounting the possibility of indirect transmission until proper studies have been performed to support this view. ${ }^{4,5}$ Specifically, if we are to rule out indirect transmission as a likely route, we should do so based on adequate supporting data. What data do we need? The scenario in Figure 1 illustrates the primary knowledge gaps.

\footnotetext{
Author for correspondence: M. Khalid Ijaz, E-mail: Khalid.Ijaz@rb.com

Cite this article: Ijaz MK, Nims RW, and McKinney J. (2022). Indirect transmission of severe acute respiratory syndrome coronavirus virus 2 (SARS-CoV-2): What do we know and what do we not know?. Infection Control \& Hospital Epidemiology, 43: 676-678, https://doi.org/10.1017/ice.2021.57
}

What does our current knowledge tell us about the risk of acquiring infectious SARS-CoV-2 during the scenario illustrated in Figure 1? Unfortunately, the extent of deposition of infectious SARS-CoV-2 (not viral RNA determined by PCR assay!) onto the visitor's hand when he coughs, has not been empirically determined. On the basis of the accepted respiratory droplets or aerosol transmission route, one must assume that a significant burden of infectious virus would be discharged onto the hand by such a cough. If so, why haven't the data been generated to support this? We do know that SARS-CoV-2 can survive on skin for hours (half-life of 3-5 hours at room temperature). ${ }^{6,7}$ Assuming that the SARS-CoV-2-infected visitor leaves the office within the hour, the virus deposited on his hand while coughing should remain infectious until he reaches for the door knob. Here we run into another knowledge gap, for we have no empirical data to help us assess the quantity of infectious SARS-CoV-2 that might be transferred from the visitor's hand to the door knob. Once on the steel door knob, we do, however, have empirical data to help us predict how long infectious SARS-CoV-2 can remain there. For instance, half-life data on SARS-CoV-2 survival on experimentally contaminated prototypic HITES at room temperature exist from several investigators. Reaching for the contaminated door knob as you leave the 


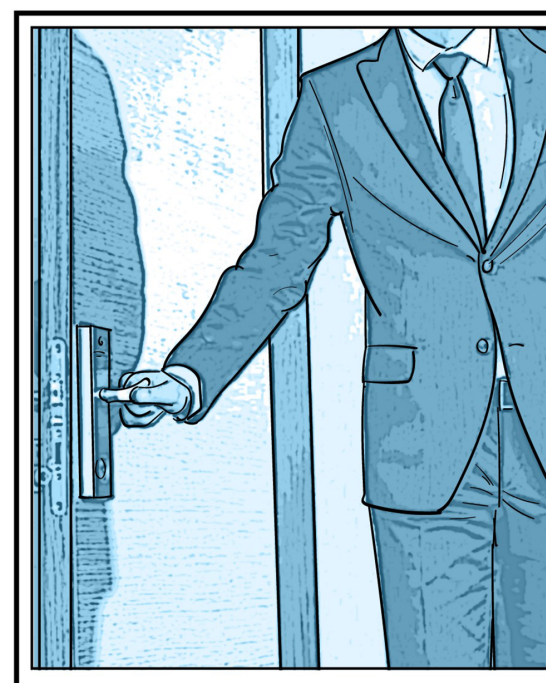

A person walks into your office...

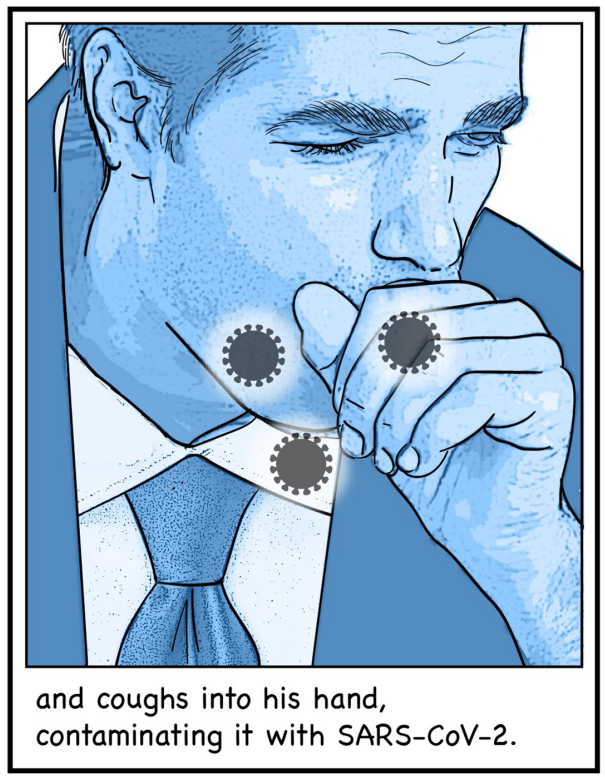

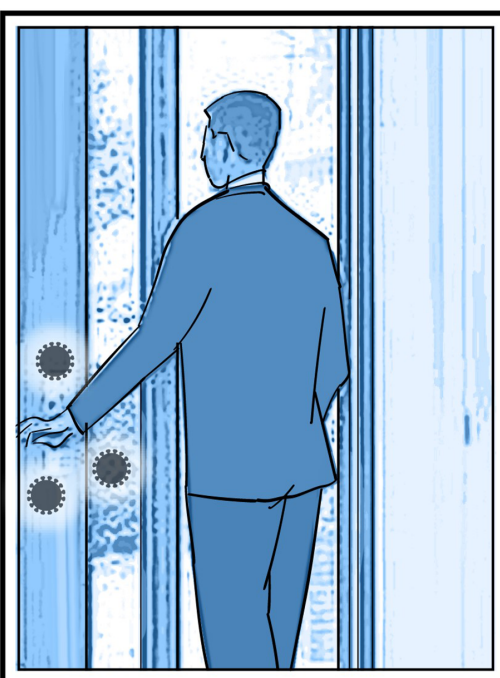

Then, he leaves the office, contaminating the door knob.

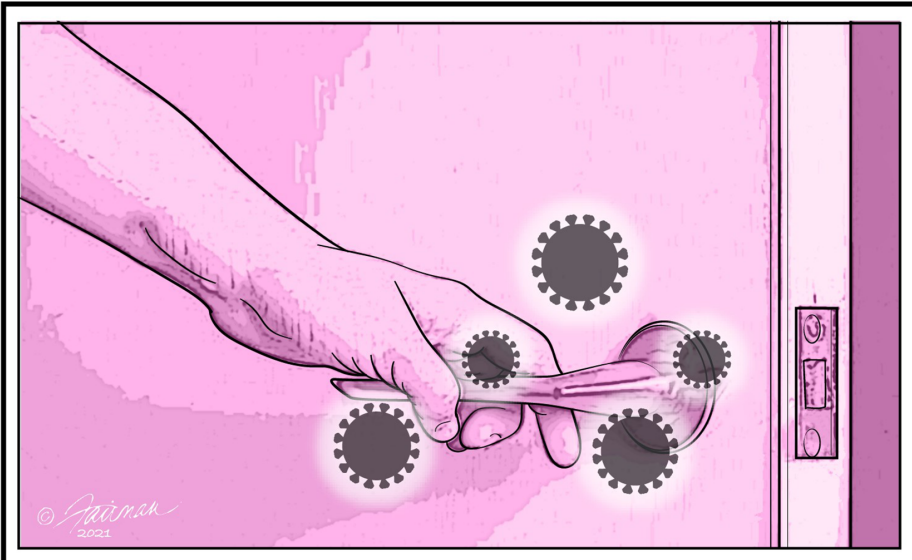

The virus sits on the door knob. You open the door, contaminating your hand...

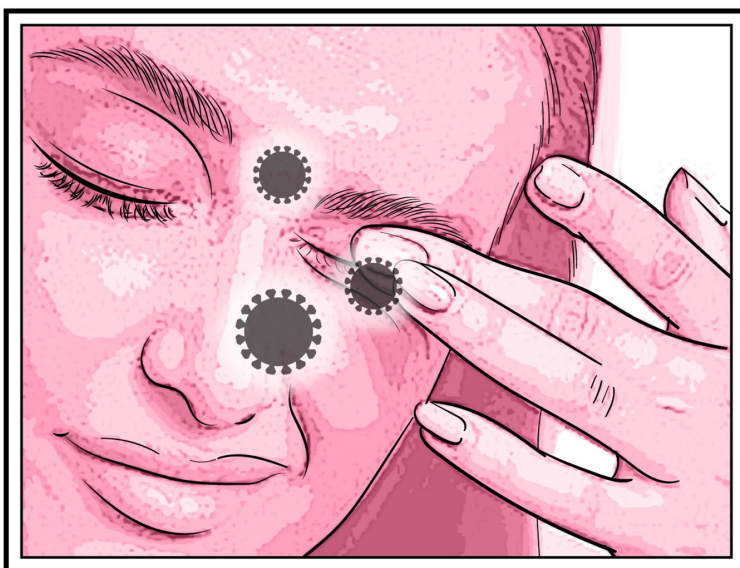

and you touch your face, contaminating your eyes and/or nose, mouth.

Fig. 1. Example scenario for an indirect transmission of SARS-CoV-2 from one person to another through virus-contaminated HITES.

office that day, there will likely be infectious SARS-CoV-2 remaining, per the half-life data for steel (19 to 143 hours at room temperature, depending on the organic matrix in which the SARS-CoV-2 was deposited). ${ }^{6,8}$ How much infectious SARS-CoV-2 will be transferred to your hand as you reach for the door knob? We do not have empirical data to allow us to assess this. Nor do we know the quantity of SARS-CoV-2 that might be transferred to your susceptible mucous membranes (ocular, nasal, oral) as you self-inoculate on the way out of the office. Finally, we simply do not yet know how much SARS-CoV-2 must be introduced to a susceptible host's mucous membranes to initiate infection (ie, the human minimal infectious dose).

For the moment, let us take the position that transmission via respiratory droplets and/or aerosols is the only relevant mode of SARS-CoV-2 transmission. Are we on a stronger footing from a data perspective? Do we, for instance, know how much infectious SARS-CoV-2 is discharged as we speak, breath, sneeze, or cough? Unfortunately, no related data are yet available. Some data on the survival of SARS-CoV-2 in aerosols have been reported, ${ }^{9}$ but the totality and quality of these data are not nearly as complete as are the surface survival data, reflecting the challenges in performing such experiments. We can use the half-life value of 1.2 hours $^{9}$ for our purposes. There is still debate over what constitutes a safe distance for avoiding possible SARS-CoV-2 transmission. It is likely $>2 \mathrm{~m},{ }^{10}$ but are we sure? Finally, we are back to the question of how much infectious SARS-CoV-2 must be introduced into a mucous membrane via portals of entry to initiate an infection. Many knowledge gaps, therefore, apply also to the direct transmission pathway.

It is generally believed that no strong empirical data support an indirect route of infectious SARS-CoV-2 dissemination. ${ }^{1,2}$ We would like to turn this argument on its head; namely, do we have enough empirical data to rule out an indirect transmission route for infectious SARS-CoV-2? In the interest of public safety, we strongly encourage investigators to begin closing these knowledge gaps.

Acknowledgments. We acknowledge Jennifer Fairman for creating the figure. We also thank Dr Chris Jones and Dr Mark Ripley, both from Reckitt Benckiser R\&D, for their critical review of the manuscript and feedback. 
Financial support. Reckitt Benckiser funded the preparation of the manuscript and the figure.

Conflicts of interest. Raymond Nims received a fee from Reckitt Benckiser for writing and editing the manuscript. The other authors report no conflicts of interest relevant to this article.

\section{References}

1. Goldman E. Exaggerated risk of transmission of COVID-19 by fomites. Lancet Infect Dis 2020;20:892-893.

2. Meyerowitz EA, Richterman A, Gandhi RT, et al. Transmission of SARSCoV-2: a review of viral, host, and environmental factors. Ann Int Med 2020. doi: 10.7326/M20-5008.

3. Transmission of SARS-CoV-2: implications for infection prevention precautions. World Health Organization website. https://www.who.int/newsroom/commentaries/detail/transmission-of-sars-cov-2-implications-forinfection-prevention-precautions. Published 2020. Accessed February 8, 2021.

4. Cai J, Sun W, Huang J, et al. Indirect virus transmission in cluster of COVID-19 cases, Wenzhou, China, 2020. Emerg Inf Dis 2020;26:1343-1345.
5. Kanamori H. Rethinking environmental contamination and fomite transmission of SARS-CoV-2 in the healthcare. J Infect 2020. doi: 10.1016/j. jinf.2020.08.041

6. Hirose R, Ikegaya $\mathrm{H}, \mathrm{Naito} \mathrm{Y}$, et al. Survival of severe acute respiratory syndrome coronavirus (SARS-CoV-2) and influenza virus on human skin: importance of hand hygiene in coronavirus disease 2019 (COVID-19). Clin Infect Dis 2020. doi: 10.1093/cid/ciaa1517.

7. Harbourt DE, Haddow AD, Piper AE, et al. Modeling the stability of severe acute respiratory syndrome coronavirus 2 (SARS-CoV-2) on skin, currency, and clothing. PLOS Negl Trop Dis 2020;14(11):e0008831.

8. Ijaz MK, Nims RW, Zhou SS, et al. Microbicidal actives with virucidal efficacy against SARS-CoV-2 and other beta- and alpha-coronaviruses and implications for future emerging coronaviruses and other enveloped viruses. Sci Rep 2021, In press.

9. van Doremalen N, Morris DH, Holbrook MG, et al. Aerosol and surface stability of SARS-CoV-2 as compared with SARS-CoV-1. N Engl J Med 2020;382:1564-1567.

10. Setti L, Passarini F, De Gennaro G, et al. Airborne transmission route of COVID-19: why 2 meters/ 6 feet of inter-personal distance could not be enough. Int J Res Public Health 2020;17(8):2932.

\title{
Could it be that the B.1.1.7 lineage is more deadly?
}

\author{
Chia Siang Kow ${ }^{1}$ and Syed Shahzad Hasan ${ }^{2,3}$ \\ ${ }^{1}$ School of Postgraduate Studies, International Medical University, Kuala Lumpur, Malaysia, ${ }^{2}$ School of Applied Sciences, University of Huddersfield, Huddersfield, \\ United Kingdom and ${ }^{3}$ School of Biomedical Sciences \& Pharmacy, University of Newcastle, Callaghan, Australia
}

To the Editor-The world had been shocked by the emergence of a new variant (B.1.1.7) of severe acute respiratory syndrome coronavirus 2 (SARS-CoV-2), which might have been circulated since September 2020 from the southeastern region of England. ${ }^{1}$ As reported, this new lineage of SARS-CoV-2 has acquired 17 mutations in its genome that lead to amino acid changes within the Spike receptor-binding domain. ${ }^{1}$ The analyses thus far have indicated that the B.1.1.7 lineage might be more transmissible than other SARS-CoV-2 lineages, with a reproduction ratio higher than those of other SARS-CoV-2 lineages by 0.4 and 0.7 (ie, up to $70 \%$ more transmissible). ${ }^{2}$ Santos et $\mathrm{al}^{3}$ employed in silico methods to analyze the interaction between the Spike receptor-binding domain of the B.1.1.7 variant and the ACE2 receptor. They discovered that the N501Y mutant residue on the spike protein of the B.1.1.7 variant establishes a more significant number of interactions with the ACE2 receptor, indicating an increased interaction force with the ACE2 receptor, which could explain its increased infectivity. In contrast, although the newly discovered 501Y.V2 variant, which spread rapidly in the Eastern Cape and Western Cape Provinces of South Africa, also contains the N501Y mutant residue on the spike protein, the substitutions $\mathrm{K} 417 \mathrm{~N}$ and $\mathrm{E} 484 \mathrm{~K}$ in the South African variant $501 . \mathrm{V} 2$ would reduce its binding affinity with ACE2 receptor, resulting in binding affinity comparable to that of the wild-type Spike receptor-binding domain. ${ }^{4}$

Arif $^{5}$ commented that there is uncertainty regarding the severity of disease in people infected with SARS-CoV-2 of the

\footnotetext{
Author for correspondence: Chia Siang Kow, E-mail: chiasiang_93@hotmail.com

Cite this article: Kow CS and Hasan SS. (2022). Could it be that the B.1.1.7 lineage is more deadly?. Infection Control \& Hospital Epidemiology, 43: 678-679, https://doi.org/ $10.1017 /$ ice.2021.59
}

B.1.1.7 lineage. Nevertheless, the general assumption that the B.1.1.7 lineage would not lead to increased severity of COVID19 may not hold true because increased binding affinity between the Spike receptor-binding domain and ACE2 receptor could lead to more ACE2 downregulation should an individual acquire this new variant compared to other variants. ${ }^{6}$ Interestingly, the mutation in the D614G variant, which currently dominates in much of the world, does not increase Spike protein affinity for ACE2.? Indeed, the D614G variant is not associated with increased severity of COVID-19 compared to the ancestral strain, although with increased viral load. ${ }^{8,9}$ Until the association between B.1.1.7 lineage and increased severity of COVID-19 is conclusively discredited, perhaps patients who acquire the new B.1.1.7 variant should be managed more aggressively with anti-inflammatory therapies, and the current antiviral armamentarium of COVID-19, especially remdesivir, should be evaluated if it preserves its efficacy against this new variant.

\section{Acknowledgments.}

Financial support. No financial support was provided relevant to this article.

Conflicts of interest. All authors report no conflicts of interest relevant to this article.

\section{References}

1. Kupferschmidt K. Fast-spreading UK virus variant raises alarms. Science 2021;371(6524):9-10.

2. Volz E, Mishra S, Chand M, et al. Transmission of SARS-CoV-2 lineage B.1.1.7 in England: insights from linking epidemiological and genetic data. medRxiv 2020. doi: 10.1101/2020.12.30.20249034. 